\title{
Hydrodynamic Description of Heavy Ion Collisions
}

\author{
Chiho Nonaka \\ Department of Physics, Nagoya University, Nagoya 464-8602, Japan \\ E-mail: nonaka@hken.phys.nagoya-u.ac.jp
}

\begin{abstract}
We give a short review of hydrodynamic models at heavy ion collisions from the point of view of initial conditions, an equation of states (EoS) and freezeout process. Then we show our latest results of a combined fully three-dimensional macroscopic/microscopic transport approach. In this model for the early, dense, deconfined stage relativistic 3D-hydrodynamics of the reaction and a microscopic nonequilibrium model for the later hadronic stage where the equilibrium assumptions are not valid anymore are employed. Within this approach we study the dynamics of hot, bulk QCD matter, which is being created in ultra-relativistic heavy ion collisions at RHIC.
\end{abstract}

\section{Hydrodynamic Models at RHIC}

The first five years of RHIC operations at $\sqrt{s_{N N}}=130 \mathrm{GeV}$ and $\sqrt{s_{N N}}=200 \mathrm{GeV}$ have yielded a vast amount of interesting and sometimes surprising results. There exists mounting evidence that RHIC has created a hot and dense state of deconfined QCD matter with properties similar to that of an ideal fluid [1, 2] - this state of matter has been termed the strongly interacting Quark-Gluon-Plasma (sQGP). One of the evidence is the success of ideal hydrodynamic models in various physical observables. Especially, for the first time, in elliptic flow the hydrodynamic limit shows good agreement with the experimental data at RHIC, though at AGS and SPS hydrodynamic models give larger value as compared to experimental data.

The sophisticated 3D ideal hydrodynamic calculations, however, reveal that many of experimental data have not yet been fully evaluated or understood [5]. For example, elliptic flow at forward and backward rapidity $₫$ and at peripheral collisions is overestimated by ideal hydrodynamical models. The Hanbury-Brown Twiss (HBT) puzzle is not completely understood from the point of view of hydrodynamics. These distinctions between hydrodynamic calculation and experimental data suggest that the ideal hydrodynamic picture is not applicable to all physical observables even in low transverse momentum region. At present our main interest in hydrodynamic models is the following: How perfect the $s Q G P$ is?

$\ddagger$ Brazil group shows improved results of $v_{2}$ at forward/backward rapidity by using event-by-event fluctuated initial conditions [3]. 
Here, first, we shall give a short review of hydrodynamic models at heavy ion collisions. In addition to a numerical procedure for solving the relativistic hydrodynamic equation, hydrodynamic models are characterized by initial conditions, EoSs and freezeout process.

The necessity of input of initial conditions for hydrodynamic models is one of the largest limitations of them. Because an initial condition is not be able to determined in the framework of hydrodynamic model itself, usually a parametrization of energy density and baryon number density based on Glauber type is used and parameters in it are determined by comparison with experimental data [4, 5, 11]. Recently there are some studies in which more basic approaches, Color Glass Condensate (CGC) [6], pQCD + saturation model [7] are used for construction of initial conditions.

The most important advantage of hydrodynamic models is that it directly incorporates an EoS as input and thus is so far the only dynamical model in which a phase transition can explicitly be incorporated. In the ideal fluid approximation - and once an initial condition has been specified - the EoS is the only input to the equations of motion and relates directly to properties of the matter under consideration. In this sense a hydrodynamic model is a bridge between QCD theory and experimental data and indispensable to describe heavy ion physics. However in usual practical hydrodynamic simulations, an EoS with 1st order phase transition (Bag model) is used. In fact, there are few studies on effect of order of QCD phase transition on physical observables [8].

Conventional hydrodynamic calculations need to assume a freezeout temperature at which the hydrodynamic evolution is terminated and a transition from the zero mean-free-path approximation of a hydrodynamic approach to the infinite mean-freepath of free streaming particles takes place. The freezeout temperature usually is a free parameter which can be fitted to measured hadron spectra. There are several approaches for dealing with freezeout process: chemical equilibrium [4, 8], partial chemical equilibrium [5], continuous emission model [9] and construction of a hybrid model of a hydro + cascade model [10, 11, 12].

In Tab. 1 several hydrodynamic models are listed from the viewpoint of initial conditions and freezeout processes, because almost the same EoS with strong 1st order phase transition is used. Reference [4] presents the first calculation which shows the remarkable agreement with experimental data for both of $P_{T}$ spectra and $v_{2}$ at RHIC. However it turns out that the assumption of single freezeout temperature where chemical freezeout and kinetic freezeout occur at the same time fails in reproducing hadron ratios correctly. To obtain correct proton $P_{T}$ spectra, we need to renormalize the $P_{T}$ spectra using the $p$ to $\pi$ ratio at the critical temperature. In addition, recently, Hirano and Gyullasy point out that the good agreement of elliptic flow with experimental data may accidentally happen. Hirano and Kolb et al. propose that the introduction of two kinds of freezeout processes, chemical freezeout and kinetic freezeout to hydrodynamic models. In this model, normalization of the $P_{T}$ spectra for each particle are obtained correctly [5], but to get better agreement with experiments in elliptic flow additional initial transverse flow is needed. At present, the combination of Gluaber type and a 
cascade model gives us the most promising result for both of $P_{T}$ spectra and $v_{2}$. Hirano et al. perform calculations using CGC for initial condition and a cascade model for freezeout process, which suggests that viscosity is not negligible even at early stage of the expansion. In the next section we show our latest results [11] based on the full 3D hydrodynamic approach 13 with the microscopic Ultra-relativistic QuantumMolecular-Dynamics (UrQMD) model [14].

Table 1. Hydrodynamic models at RHIC.

\begin{tabular}{c|l|l}
\hline Reference & Initial Conditions & Freezeout Process \\
\hline$[4]$ & Glauber type & chemical equilibrium \\
\hline$[5]$ & Glauber type & partial chemical equilibrium \\
\hline$[10,11]$ & Glauber type & cascade model \\
\hline$[15]$ & CGC & partial chemical equilibrium \\
\hline$[12]$ & CGC & cascade model \\
\hline
\end{tabular}

\section{Hydro+UrQMD Model}

We calculate hadron distribution at switching temperature from the 3D hydrodynamic model using Cooper-Frye formula [16] and produce initial conditions for UrQMD model by Monte Carlo from it. Such hybrid macro/micro transport calculations are to date the most successful approaches for describing the soft physics at RHIC. The biggest advantage of the hydrodynamic description is that it directly incorporates an EoS as input - one of its largest limitations is that it requires thermalized initial conditions and one is not able to do an ab-initio calculation.

Figure 1 shows a schematic sketch of the full 3D hydrodynamic model + UrQMD. After heavy ion collisions, first, hydrodynamic expansion starts. We introduce one more parameter, switching temperature from hydrodynamic picture to hadron base event generator, UrQMD. This switching temperature should be just below the critical temperature. Here it is set to $160 \mathrm{MeV}$.

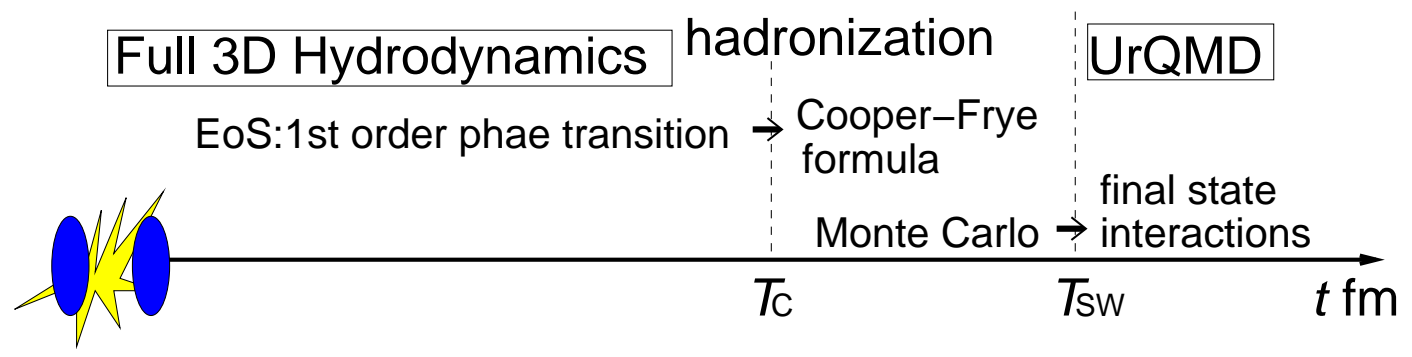

Figure 1. Schematic sketch of $3 \mathrm{D}$ hydro+UrQMD model. $T_{\mathrm{c}}(=160 \mathrm{MeV})$ and $T_{\mathrm{SW}}(=$ 150) $\mathrm{MeV}$ are critical temperature and switching temperature from hydrodynamics to UrQMD model, respectively. 
Figure 2 shows the $P_{T}$ spectra of $\pi^{+}, K$ and $p$ at $\sqrt{s_{N N}}=200 \mathrm{GeV}$ central collisions. The most compelling feature is that the hydro+micro approach is capable of accounting for the proper normalization of the spectra for all hadron species without any additional correction as is performed in the pure hydrodynamic model. The introduction of a realistic freezeout process provides therefore a natural solution to the problem of separating chemical and kinetic freeze-out in a pure hydrodynamic approach.

In Fig. 3 centrality dependence of $P_{T}$ spectra of $\pi^{+}$is shown. The impact parameter for each centrality is determined simply by the collision geometry. The separation between model results and experiment appears at lower transverse momentum in peripheral collisions compared to central collisions, just as in the pure hydrodynamic calculation. The 3D hydro + micro model does not provide any improvement for this behavior, since the hard physics high $P_{T}$ contribution to the spectra occurs at early reaction times before the system has reached the QGP phase and is therefore neither included in the pure 3D hydrodynamic calculation nor in the hydro+micro approach.

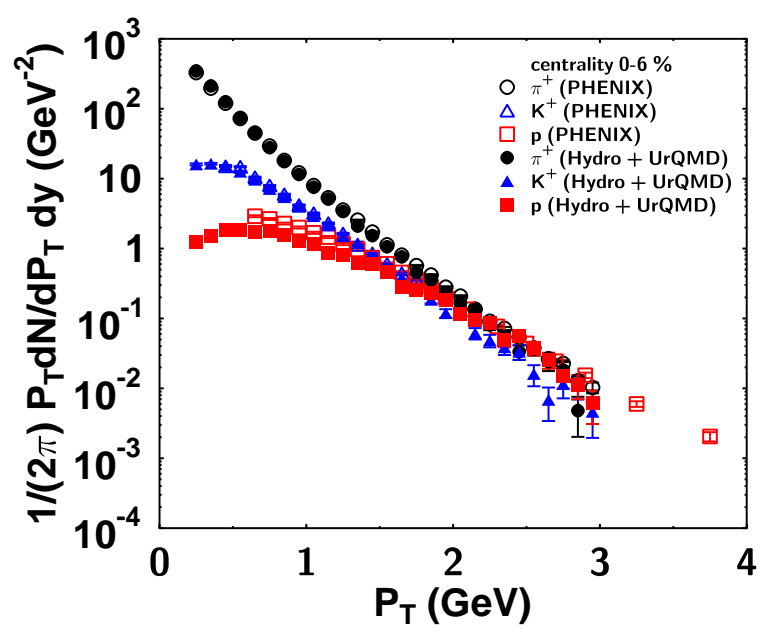

Figure 2. $P_{T}$ spectra for $\pi^{+}$, $K^{+}$and $p$ at central collisions with PHENIX data [17].

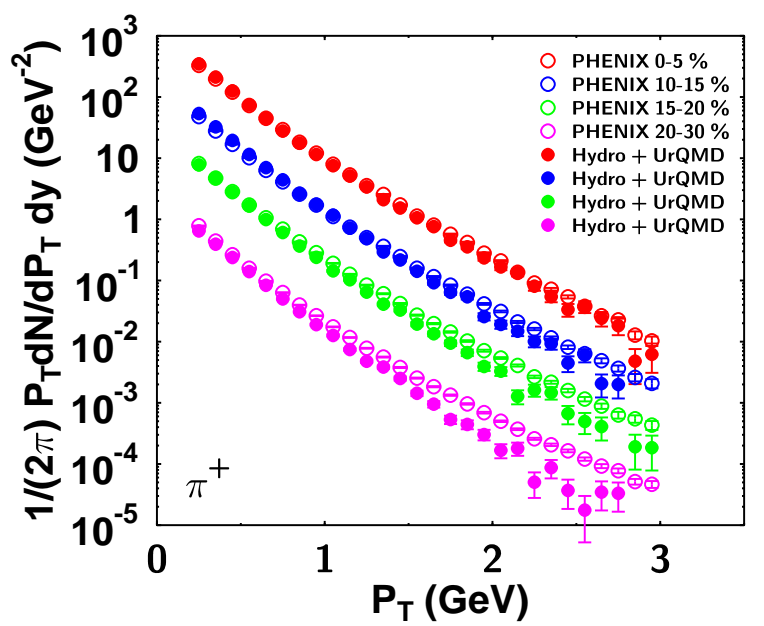

Figure 3. Centrality dependence of $P_{T}$ spectra of $\pi^{+}$with PHENIX data [17]. The $P_{T}$ spectra at $10-15$ $\%, 15-20 \%$ and $20-30 \%$ are divided by 5,25 and 200 , respectively.

Figure 4 shows the centrality dependence of the pseudorapidity distribution of charged hadrons compared to PHOBOS data [18]. Solid circles stand for model results and open circles denote data taken by the PHOBOS collaboration [18]. The impact parameters are set to $b=2.4,4.5,6.3,7.9$ fm for 0-6\%, 6-15\%, 15-25\% and 25$35 \%$ centralities, respectively. Our results are consistent with experimental data over a wide pseudorapidity region. There is no distinct difference between 3-D ideal hydrodynamic model and the hydro + UrQMD model in the centrality dependence of the psuedorapidity distribution, indicating that the shape of psuedorapidity distribution is insensitive to the detailed microscopic reaction dynamics of the hadronic final state 
[11].

In Fig. 5 we analyze the $P_{T}$ spectra of multistrange particles. Our results show good agreement with experimental data for $\Lambda, \Xi, \Omega$ for centralities $0-5 \%$. In this calculation the additional procedure for normalization is not needed (Fig. 6). Recent experimental results suggest that at thermal freezeout multistrange baryons exhibit less transverse flow and a higher temperature closer to the chemical freezeout temperature compared to non- or single-strange baryons [19, 20]. This behavior can be understood in terms of the flavor dependence of the hadronic cross section, which decreases with increasing strangeness content of the hadron. The reduced cross section of multi-strange baryons leads to a decoupling from the hadronic medium at an earlier stage of the reaction, allowing them to provide information on the properties of the hadronizing QGP less distorted by hadronic final state interactions

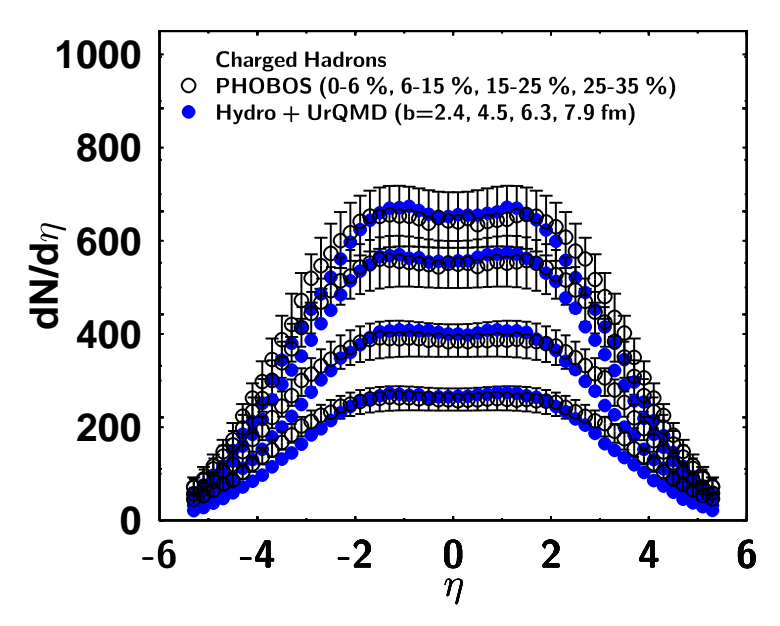

Figure 4. Centrality dependence of pseudorapidity distribution of charged particles with PHOBOS data [18.

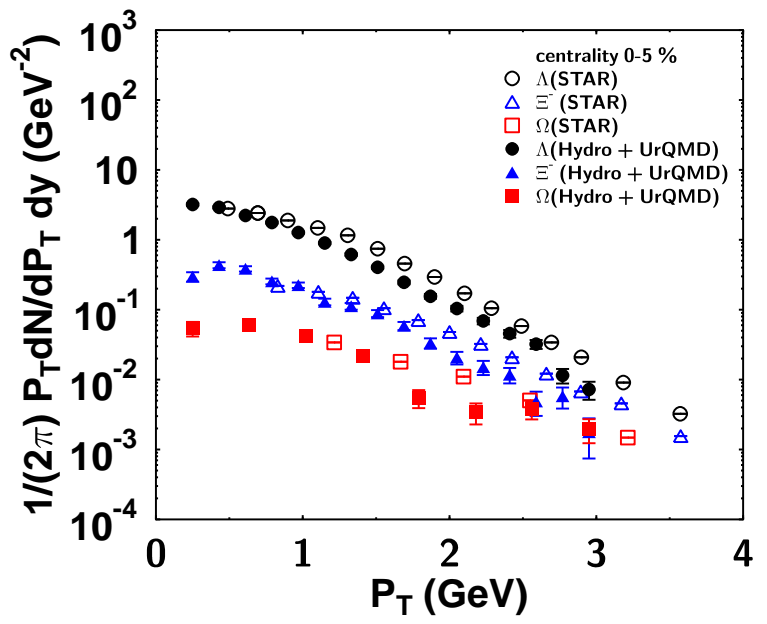

Figure 5. $P_{T}$ spectra of multistrange particles at centralities 0 $5 \%$ and $10-20 \%$ with STAR data [19.

In Fig. 7 the mean transverse momentum $\left\langle P_{T}\right\rangle$ as a function of hadron mass is shown. Open symbols denote the value at $T_{\mathrm{sw}}=150 \mathrm{MeV}$, corrected for hadronic decays. Not surprisingly, in this case the $\left\langle P_{T}\right\rangle$ follow a straight line, suggesting a hydrodynamic expansion. However if hadronic rescattering is taken into account (solid circles) the $\left\langle P_{T}\right\rangle$ do not follow the straight line any more: the $\left\langle P_{T}\right\rangle$ of pions is actually reduced by hadronic rescattering (they act as a heat-bath in the collective expansion), whereas protons actually pick up additional transverse momentum in the hadronic phase. RHIC data by the STAR collaboration is shown via the solid triangles - overall the proper treatment of hadronic final state interactions significantly improves the agreement of the model calculation with the data.

In Fig. 8 we plot elliptic flow $v_{2}$ as a function of $P_{T}$. The solid line stands for the pure hydro calculation, terminated at the switching temperature $T_{\mathrm{sw}}$ and solid circles 


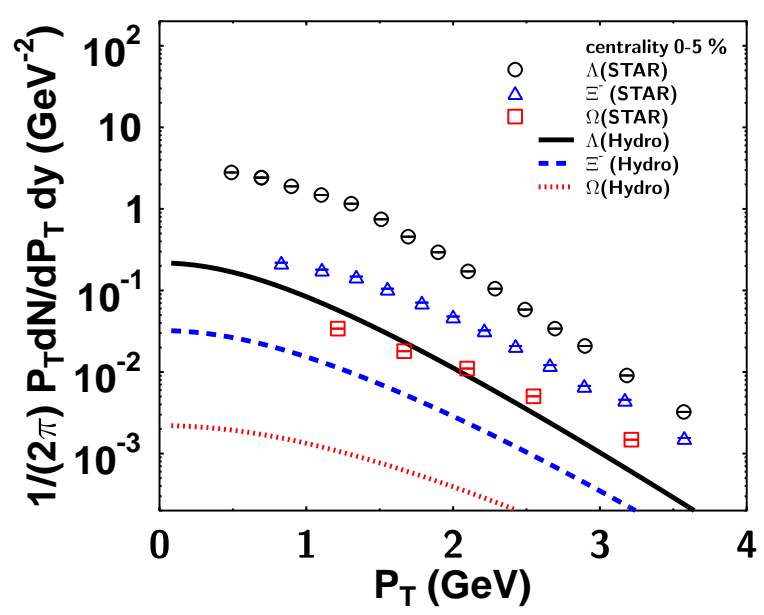

Figure 6. $P_{T}$ spectra for multistrange baryons at central collisions from pure hydro with STAR data [19.

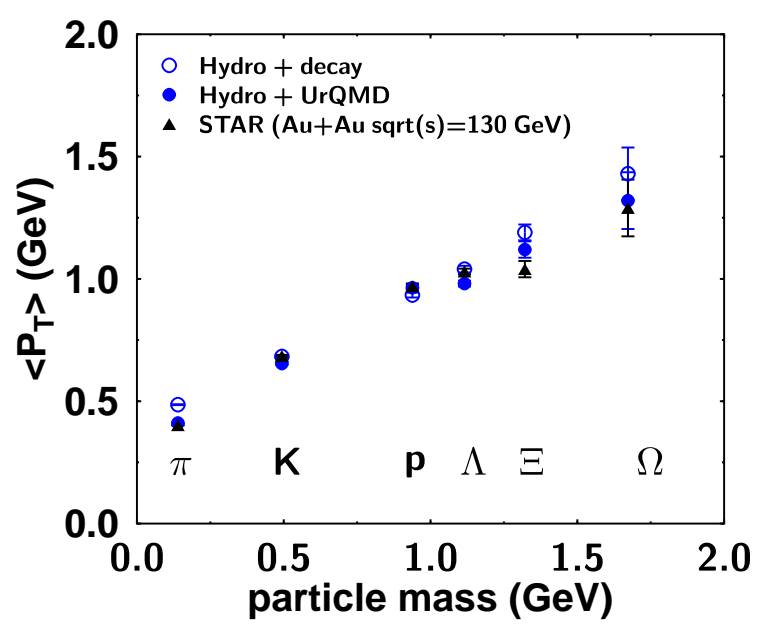

Figure 7. Mean $P_{T}$ as a function of mass with STAR data $(\mathrm{Au}+\mathrm{Au}$ $\sqrt{s_{N N}}=130 \mathrm{GeV}$ ) [20].

denote the full hydro+micro calculation. We find that the QGP contribution to the elliptic flow depends on the transverse momentum - for low $P_{T}$ nearly $100 \%$ of the elliptic flow is created in the QGP phase of the reaction, whereas the hadronic phase contribution increases to $25 \%$ at a $P_{T}$ of $1 \mathrm{GeV} / c$.

Figure 9 shows the elliptic flow as a function of $\eta$ : the pure hydrodynamic calculation is shown by the solid curve, the hydrodynamic contribution at $T_{\mathrm{sw}}$ is denoted by the dashed line and the full hydro+micro calculation is given by the solid circles, together with PHOBOS data (solid triangles). The shape of the elliptic flow in the pure hydrodynamic calculation at $T_{\mathrm{sw}}$ is quite different from that of the full hydrodynamic one terminated at a freeze-out temperature of $110 \mathrm{MeV}$. Apparently the slight bump at forward and backward rapidities observed in the full hydrodynamic calculation develops first in the later hadronic phase, since it is not observed in the calculation terminated at $T_{\mathrm{sw}}$. Evolving the hadronic phase in the hydro+micro approach will increase the elliptic flow at central rapidities, but not in the projectile and target rapidity domains. As a result, the elliptic flow calculation in the hydro+micro approach is closer to the experimental data when compared to the pure hydrodynamic calculation.

We developed a novel implementation of the well known hybrid macroscopic/microscopic transport approach, combining a newly developed relativistic $3+1$ dimensional hydrodynamic model for the early deconfined stage of the reaction and the hadronization process with a microscopic non-equilibrium model for the later hadronic stage.

Within this approach we have dynamically calculated the freezeout of the hadronic system, accounting for the collective flow on the hadronization hypersurface generated by the QGP expansion. We have compared the results of our hybrid model and of 


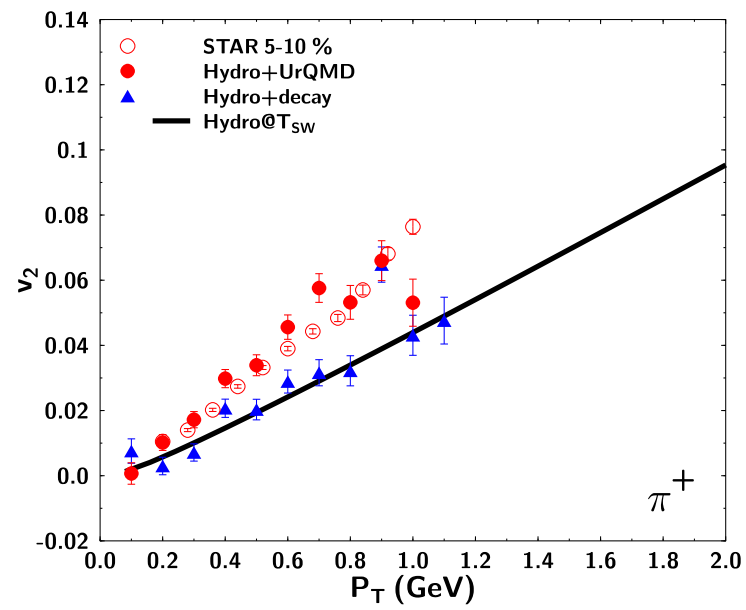

Figure 8. Elliptic flow as a function of $P_{T}$ of $\pi^{+}$at centrality $5-10 \%$ with STAR data 21 .

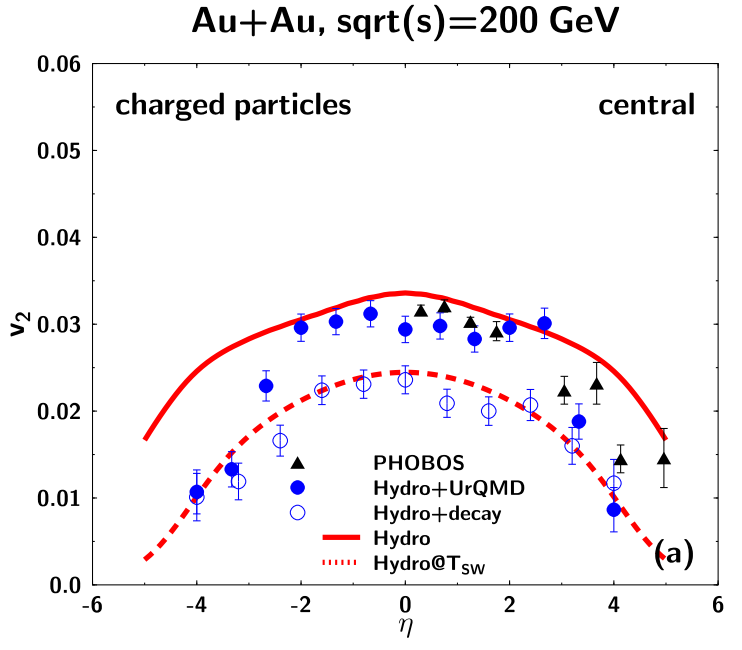

Figure 9. Elliptic flow as a function of $\eta$ of charged particles with PHOBOS data 22 .

a calculation utilizing our hydrodynamic model for the full evolution of the reaction to experimental data. This comparison has allowed us to quantify the strength of dissipative effects prevalent in the later hadronic phase of the reaction, which cannot be properly treated in the framework of ideal hydrodynamics.

\section{Summary}

The full 3D relativistic hydrodynamics + cascade model is one of successful and realistic models for description of dynamics of hot QCD bulk matter at RHIC, which helps us to understand medium property at RHIC in detail. Using this model, we can explore interesting phenomena which are caused by interactions between medium and jets [23, 24]. One of proposed interesting physical observables is mach cone [24] for the wake of them, from which we can know medium property in detail, too.

However still further investigation for EoS and initial conditions in hydrodynamics is needed. Especially the EoS is the key to know the QCD phase transition directly from comparison with experimental data. Recent lattice QCD calculation shows relatively high critical temperature with crossover phase transition and the existence of critical end point [25], which suggests that the EoS with strong 1st phase transition is not realistic. Becuase in hydrodynamic calculations, however, outputs may also be changed easily by choice of initial conditions and freezeout process, which makes it difficult to obtain the conclusive discussion on the EoS. First of all, we have to determine the most realistic initial conditions and freezeout process in hydrodynamic models before a detailed discussion about the EoS.

Furthermore, viscosity effect in medium also starts to be discussed actively [26]. Due to serious difficulty in construction not only of a viscous hydrodynamic code 
but also of framework of viscous hydrodynamics without the causality problem, the progress of study of viscous hydrodynamics has been slow. However, by virtue of recent rapid development, the practical calculations with viscous hydrodynamics which are comparable to experimental data will be achieved in the near future [26].

Finally, at LHC heavy ion collisions experiments will start in a year. Upcoming LHC data shall bring a lot of interesting, fruitful and even unexpected results for QGP physics, where hydrodynamic description will be helpful and useful for understanding of it.

\section{Acknowledgment}

We would like to thank Steffen A. Bass, Berndt Muller, Masayuki Asakawa and Joseph Kapusta for many valuable discussions and encouragement in pursuit of this work. The work is supported by Saneyoshi Shougakukai and the 21st century COE "The Origin of the Universe and Matter: Physical Elucidation of the Cosmic History" program of Nagoya University.

\section{References}

[1] T. Ludlam, Nucl. Phys. A 750, 9 (2005).

[2] M. Gyulassy and L. McLerran, Nucl. Phys. A 750, 30 (2005).

[3] R. Andrade et al., Phys. Rev. Lett. 97, 202302 (2006).

[4] P. F. Kolb, P. Huovinen, U. Heinz, H. Heselberg, Phys. Lett. B500, 232 (2001); P. Huovinen, P. F. Kolb, U. Heinz, P. V. Ruusukanen, S. A. Voloshin, Phys. Lett. B503, 58 (2001).

[5] T. Hirano and K. Tsuda, Phys. Rev. C66, 054905 (2002); P. F. Kolb, R. Rapp, Phys. Rev. C67, 044903 (2003).

[6] T. Hirano, these proceedings; R. Fries, these proceedings.

[7] K. J. Eskola et al., Phys. Rev. C72, 044904 (2005).

[8] P. Huovinen, Nucl. Phys. A761, 29 (2005).

[9] F. Grassi, Y. Hama and T. Kodama, Phys. Lett. 355, 9 (1995); Z. Phys. C73, 153 (1996).

[10] S. A. Bass, A. Dumitru, Phys. Rev. C61, 064909 (2000); D. Teaney, J. Lauret,E. V. Shuryak, Phys. Rev. Lett. 86, 4783 (2001); nucl-th/0110037.

[11] C. Nonaka, S. A. Bass, Phys. Rev. C75 014902 (2007).

[12] T. Hirano, U. Heinz, D. Kharzeev, R. Lacey, Y. Nara, Phys. Lett. B636, 299 (2006).

[13] C. Nonaka, E. Honda, S. Muroya, Eur. Phys. J. C17, 663 (2000).

[14] S. A. Bass et al., Progr. Part. Nucl. Physics Vol. 41, 225 (1998) Progr. Part. Nucl. Physics Vol. 41, 225 (1998); M. Bleicher et al., J. Phys. G25, 1859 (1999).

[15] T. Hirano, Y. Nara, Nucl. Phys. A743, 305 (2004).

[16] F. Cooper and G. Frye, Phys. Rev. D10, 186 (1974).

[17] S. S. Adler et al. (PHENIX Collaboration), Phys. Rev. C69, 034909 (2004).

[18] B. B. Back et al. (PHOBOS Collaboration), Phys. Rev. Lett. 91, 052303 (2003).

[19] M. Estienne (for the STAR Collaboration), J. Phys. G31, S873 (2005).

[20] J. Adames et al. (STAR Collaboration), Phys. Rev. Lett. 92, 182301 (2004).

[21] J. Adams et al. (STAR Collaboration), Phys. Rev. C72, 014904 (2005).

[22] B. B. Back et al. (PHOBOS Collaboration), Phys. Rev. C72, 051901(R) (2005).

[23] T. Renk, J. Ruppert, C. Nonaka, S. A. Bass, nucl-th/0611027, to appear in Phys. Rev. C.

[24] Casalderrey-Solana, these proceedings; T. Renk, these proceedings.

[25] T. Hatsuda, these proceedings.

[26] D. Teaney, these proceedings; T. Kodama, these proceedings; A. Muronga, nucl-th/0611090, nucl-th/0611091; U. Heinz, H. Song, A. K. Chaudhuri, Phys. Rev. C73,034904,2006. 